\title{
SU(N)Heisenberg model with multicolumn representations
}

$\operatorname{AUTHOR}(\mathrm{S})$ :

Okubo, Tsuyoshi; Harada, Kenji; Lou, Jie;

Kawashima, Naoki

\section{CITATION:}

Okubo, Tsuyoshi ... [et al]. SU(N)Heisenberg model with multicolumn representations. Physical Review B 2015, 92(13): 134404.

\section{ISSUE DATE:}

2015-10-05

URL:

http://hdl.handle.net/2433/200788

RIGHT:

C)2015 American Physical Society 
PHYSICAL REVIEW B 92, 134404 (2015)

\title{
$\mathrm{SU}(N)$ Heisenberg model with multicolumn representations
}

\author{
Tsuyoshi Okubo, ${ }^{1, *}$ Kenji Harada, ${ }^{2}$ Jie Lou, ${ }^{3}$ and Naoki Kawashima ${ }^{1}$ \\ ${ }^{1}$ Institute for Solid State Physics, University of Tokyo, Kashiwa, Chiba 277-8581, Japan \\ ${ }^{2}$ Graduate School of Informatics, Kyoto University, Kyoto 606-8501, Japan \\ ${ }^{3}$ Department of Physics, Fudan University, Shanghai 200433, China
}

(Received 21 April 2015; revised manuscript received 7 September 2015; published 5 October 2015)

\begin{abstract}
The $\mathrm{SU}(N)$ symmetric antiferromagnetic Heisenberg model with multicolumn representations on the twodimensional square lattice is investigated by quantum Monte Carlo simulations. For the representation of a Young diagram with two columns, we confirm that a valence-bond solid (VBS) order appears as soon as the Néel order disappears at $N=10$, indicating no intermediate phase. In the case of the representation with three columns, there is no evidence for either the Néel or the VBS ordering for $N \geqslant 15$. This is actually consistent with the large- $N$ theory, which predicts that the VBS state immediately follows the Néel state, because the expected spontaneous order is too weak to be detected.
\end{abstract}

DOI: 10.1103/PhysRevB.92.134404

PACS number(s): $75.10 . J \mathrm{~m}, 75.40 . \mathrm{Mg}$

\section{INTRODUCTION}

Realization of quantum spin liquid in short-range coupling models has been a popular research target in condensed-matter physics for several decades. One approach to obtain a spinliquid state is to consider a Hamiltonian with higher symmetry, which increases quantum fluctuations. Read and Sachdev generalized the antiferromagnetic Heisenberg into $\mathrm{SU}(N)$ symmetry $[1,2]$. Based on the $1 / N$ expansion they showed that the ground state of the model with sufficiently large $N$ is a valence-bond-solid (VBS) ordering breaking the lattice rotational or the translational symmetry spontaneously. Recently, in terms of the deconfined quantum criticality [3-5], their theory attracts much attention. In particular, the existence of an intermediate state, which might be a spin-liquid state, was discussed near the boundary of Néel and VBS [6-8].

The nature of the ground states of the model can vary depending on the representation of $\operatorname{SU}(N)$ algebra, which is determined through an $m$ rows and $n$ columns Young diagram. The representation with the single-column $[(m, n)=(m, 1)]$ Young diagram can be understood as the system with $m$ fermions per sites, while the single-row $[(m, n)=(1, n)]$ Young diagram means $n$ bosons per site. In the case of familiar SU(2) spins, the $n$ bosons correspond to the $S=n / 2$ spin systems. Thus one can imagine that the columns in general $\mathrm{SU}(N)$ models are similar to the spin length $S$. Read and Sachdev suggested that the ground-state phase diagram on the $N-n$ plane does not strongly depend on the value of $m$. Within the $1 / N$ expansion, there are only two types of phases: the small- $N$ Néel phase and the large- $N$ VBS phase [see Fig. 1(a)]. In addition, it was shown that the nature of the VBS state can be classified by the remainder of the division of $n$ by 4 on the two-dimensional square lattice. For $n=1,3(\bmod 4)$, the VBS state is so-called columnar VBS, where both the translational symmetry and $90^{\circ}$ lattice rotational symmetry are broken [Fig. 1(b)]. For $n=2(\bmod 4)$, the VBS state is expected to be a nematic VBS with breaking only lattice rotational symmetry [Fig. 1(c)]. In the case of $n=0(\bmod 4)$, there is no spontaneous symmetry breaking, which is an analog of the
Haldane state in the $S=1$ spin chain $[9,10]$. Since the number of boxes $n$ corresponds to the spin length, Read and Sachdev's prediction is the two-dimensional version of the famous Haldane works $[9,10]$. Therefore their theory is important in the exploration of exotic states in two-dimensional systems.

Beyond the $1 / N$ expansion of Read and Sachdev's work, it was shown that for $(n, m)=(1,1)$ the ground state is the $\operatorname{SU}(N)$ Néel state for $N \leqslant 4$, while it becomes the columnar VBS state for $N \geqslant 5$ by the quantum Monte Carlo (QMC) calculation [6,7]. Related to this case, the $\mathrm{SU}(N) J-Q$ model was proposed $[11,12]$. The $\mathrm{SU}(N) J-Q$ model has an additional many-body interaction so that the quantum phase transition between the Néel phase and the columnar VBS phase occurs by continuously changing the Hamiltonian parameter. In order to vary the Hamiltonian continuously, a continuous- $N$ model was also proposed by Beach et al. [8]. Because the phase transition might be a realization of deconfined quantum criticality [3-5], the nature of these models has attracted much recent interest in condensed-matter physics [8,11-18].

For the case of $n \geqslant 2$, there were a few studies concerning the phase boundary between the Néel phase and the VBS phase. Although the Néel order was suppressed as $N$ was increased, no evidence of VBS order was found for $n=$ 2,3,4 with $m=1$ in QMC calculation up to $L=32$ for an $L \times L$ square lattice [7]. This result appeared to suggest an intermediate phase between the Néel phase and the VBS phases. However, whether the missing evidence of the VBS order for $n \geqslant 2$ is due to an intermediate spin-liquid phase or due to the extremely small (but finite) order parameter beyond numerical limitation has not been clarified up to now.

\section{MODEL}

In this paper, we investigate ground states of the $\mathrm{SU}(N)$ Heisenberg model for $n=2$ and 3 with $m=1$ by using QMC simulation. The $\mathrm{SU}(N)$ model we considered is an $\mathrm{SU}(N)$ symmetric antiferromagnetic Heisenberg model on the two-dimensional square lattice with the periodic boundary condition. The Hamiltonian of the model is given by

$$
\mathcal{H}=\frac{J}{N} \sum_{\langle i, j\rangle, i \in A} \sum_{\alpha, \beta=1}^{N} S_{i}^{\alpha \beta} \tilde{S}_{j}^{\beta \alpha},
$$




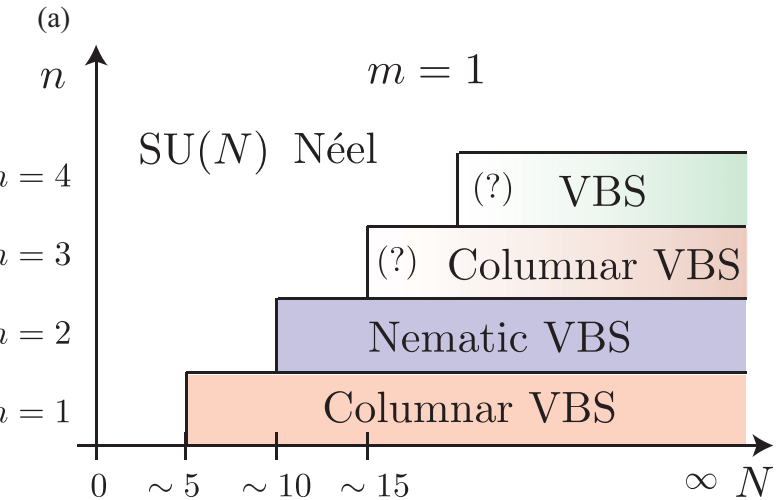

(b)

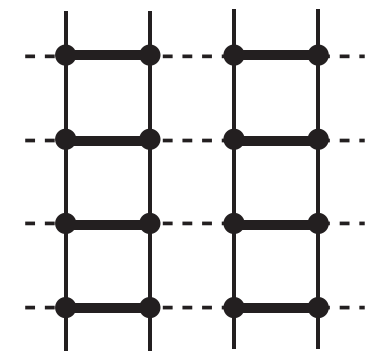

(c)

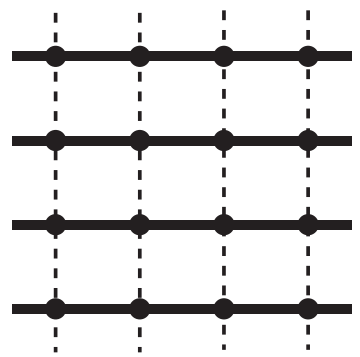

FIG. 1. (Color online) (a) Schematic phase diagram of the $\mathrm{SU}(N)$ Heisenberg model on the square lattice with single-row $(m=1)$ representations. The phase boundaries for the case of $n=2,3$ are determined in the present study, while that for $n=1$ was from Refs. [6,7]. In the case of $n=3,4$, we do not see clear evidence of the spontaneous VBS order in the vicinity of the phase boundaries for finite-size QMC simulations. (b,c) Schematic picture of the columnar VBS (b) and the nematic VBS (c) states. Thick solid lines denote the larger value of $\left\langle\sum_{\alpha, \beta}^{N} S_{i}^{\alpha \beta} \tilde{S}_{j}^{\beta \alpha}\right\rangle$, while thin solid and dashed lines indicate smaller values.

where $S_{i}^{\alpha \beta}$ and $\tilde{S}_{j}^{\beta \alpha}$ are generators of $\mathrm{SU}(N)$ algebra, and we consider $J>0$. On one sublattice $A$ of the lattice, the representation of the generators $S_{i}^{\alpha \beta}$ is characterized by the Young diagram with a single row $(m=1)$ and arbitrary number $n$ of columns, while we use the conjugate representation $\tilde{S}_{i}^{\alpha \beta}$ on the other sublattice. Note that the conjugate representation satisfies the relation $\tilde{S}_{i}^{\alpha \beta}=-S_{i}^{\beta \alpha}$. We have performed QMC simulation based on the loop algorithm. We modified the ALPS/LOOPER code $[19,20]$ for the present purpose [21]. We set the inverse temperature $\beta$ as $\beta J=L$ and investigated the zero-temperature properties by extrapolating the results to $L \rightarrow \infty$.

In order to see the VBS orders, we define two order parameters. The local nematic order parameter is defined as

$$
\Phi_{j} \equiv P_{j, y}-P_{j, x},
$$

where $P_{j, \mu}(\mu= \pm x, \pm y)$ is the nearest-neighbor product of "magnetic" moments

$$
P_{j, \mu} \equiv \sum_{\alpha=1}^{N} S_{j}^{\alpha \alpha} S_{j+\boldsymbol{e}_{\mu}}^{\alpha \alpha} .
$$

The nematic order parameter characterizes the symmetry breaking of 90 deg lattice rotation. $\left\langle\Phi_{j}\right\rangle$ takes a finite value
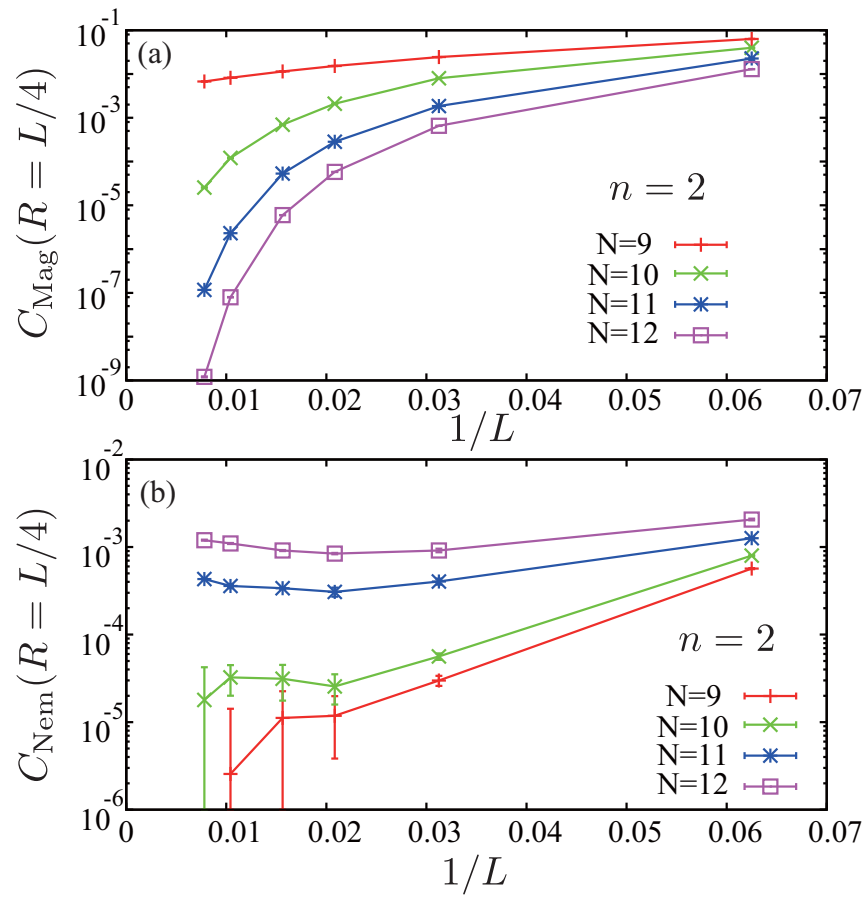

FIG. 2. (Color online) Semilog plot of the two-point correlation functions at $|\boldsymbol{R}|=L / 4$ for the model with $n=2$ as functions of $1 / L$. (a) The correlation function of the Néel order. (b) The correlation function of the nematic order. The error bars represent the standard errors.

for both the nematic VBS and the columnar VBS states in the thermodynamic limit. We also define a local complex order parameter characterizing the columnar VBS order as

$$
\Psi_{j} \equiv(-1)^{j_{x}}\left(P_{j, x}-P_{j,-x}\right)+i(-1)^{j_{y}}\left(P_{j, y}-P_{j,-y}\right),
$$

where $j_{x}$ and $j_{y}$ are integers representing the lattice coordinates of site $j$. In the columnar VBS phase $\left|\left\langle\Psi_{j}\right\rangle\right| \neq 0$, while $\left|\left\langle\Psi_{j}\right\rangle\right|=0$ for the Néel and the nematic VBS phases. In order to see the phase transition clearly, we examine the two-point correlation functions of an observable $O: C_{O}(\boldsymbol{R}) \equiv$ $\left\langle O(\mathbf{0}) O^{\dagger}(\boldsymbol{R})\right\rangle$. For the Néel order, we use the correlation of a magnetization $S_{i}^{\alpha \alpha}: C_{\mathrm{Mag}}(\boldsymbol{R}) \equiv \sum_{\alpha=1}^{N} C_{S^{\alpha \alpha}}(\boldsymbol{R})$. We also consider the nematic VBS correlation $C_{\mathrm{Nem}} \equiv C_{\Phi}(\boldsymbol{R})$ and the columnar VBS correlation $C_{\mathrm{Col}}(\boldsymbol{R}) \equiv C_{\Psi}(\boldsymbol{R})$.

\section{RESULTS}

First, we examine the case of $n=2$. In Fig. 2, we show the two-point correlations for $n=2$ at $|\boldsymbol{R}|=L / 4$ for various $N$ and system sizes $L$. For the case of the Néel order [Fig. 2(a)], we see that the correlation exponentially decays to zero by increasing $L$ for $N \geqslant 10$ while it converges to a nonzero value for $N=9$, indicating that the Néel state is the ground state for $N \leqslant 9$ and it is not for $N \geqslant 10$. These observations are consistent with the previous QMC calculation [7]. For the nematic order parameter [Fig. 2(b)], the two-point correlations tend to converge to nonzero values for $N \geqslant 10$, although the situation at $N=10$ is rather subtle because of larger statistical errors 


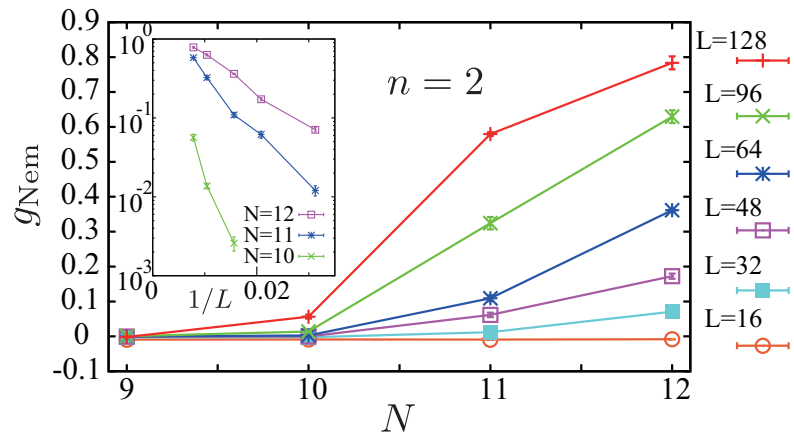

FIG. 3. (Color online) The Binder cumulant of the nematic order parameter for the model with $n=2$ for $16 \leqslant L \leqslant 128$. The inset shows size dependence of the Binder cumulant for $N=10,11$, and 12 in the semilog scale. The error bars represent the standard errors.

comparable with the correlation function itself. ${ }^{1}$ In order to confirm the appearance of the nematic VBS order at $N=10$, we plot the Binder cumulant of the nematic order parameter in Fig. 3. The Binder cumulant for the nematic order parameter is given by

$$
g_{\mathrm{Nem}} \equiv \frac{1}{2}\left(3-\frac{\left\langle\Phi^{4}\right\rangle}{\left\langle\Phi^{2}\right\rangle^{2}}\right),
$$

where $\Phi$ is the sum of local nematic order parameters: $\Phi \equiv L^{-2} \sum_{j} \Phi_{j} \cdot g_{\mathrm{Nem}}$ is normalized so that $g_{\mathrm{Nem}}=0$ for the Néel phase, while $g_{\mathrm{Nem}}=1$ for the nematic (or the columnar) VBS phase. The nematic Binder cumulant at $N=10$ develops as the system size is increased. As plotted in the inset of Fig. 3, the size dependence of the Binder cumulant at $N=10$ looks similar to those of $N=11$ and 12 , where we observed clear evidence of the nematic VBS order through the correlation function. In addition, as we see in Fig. 5, the order parameter $\sqrt{\left\langle\Phi^{2}\right\rangle}$ slightly deviates upward from the powerlaw decay, $\sqrt{\left\langle\Phi^{2}\right\rangle}(L) \propto 1 / L$, which should be obeyed asymptotically when the system is gapped. Actually, a polynomial extrapolation to the thermodynamic limit using a quadratic function of $1 / L$ leads to a finite value of the order parameter: $\langle|\Phi|\rangle \simeq 0.002$. These observations indicate the nematic VBS order at $N=10$ in the thermodynamic limit. We also checked that the order parameter $\Psi$ shows no evidence of long-range order for the case of $n=2$. From these observations, we conclude that in the case of $n=2$ the ground state is the Néel state for $N \leqslant 9$, while it is the nematic VBS state for $N \geqslant 10$. There is no intermediate phase.

Next we move to the case of $n=3$. We plot two-point correlation functions for $n=3$ at $|\boldsymbol{R}|=L / 4$ in Fig. 4. For the Néel order, we clearly see from the curvature of the curves that the Néel state is the ground state for $N \leqslant 14$ and it is not for $N \geqslant 15$. On the other hand, we do not see a clear difference among different values of $N$ in the two-point correlation function of the columnar VBS order [see Fig. 4(b)]. The behavior of the columnar VBS order

\footnotetext{
${ }^{1}$ At $N=10$ for $L \geqslant 48$, the estimated standard error is $O\left(10^{-5}\right)$, which is the same order with the mean value. Thus the converging behavior at $N=10$ is unclear within the present data set.
}
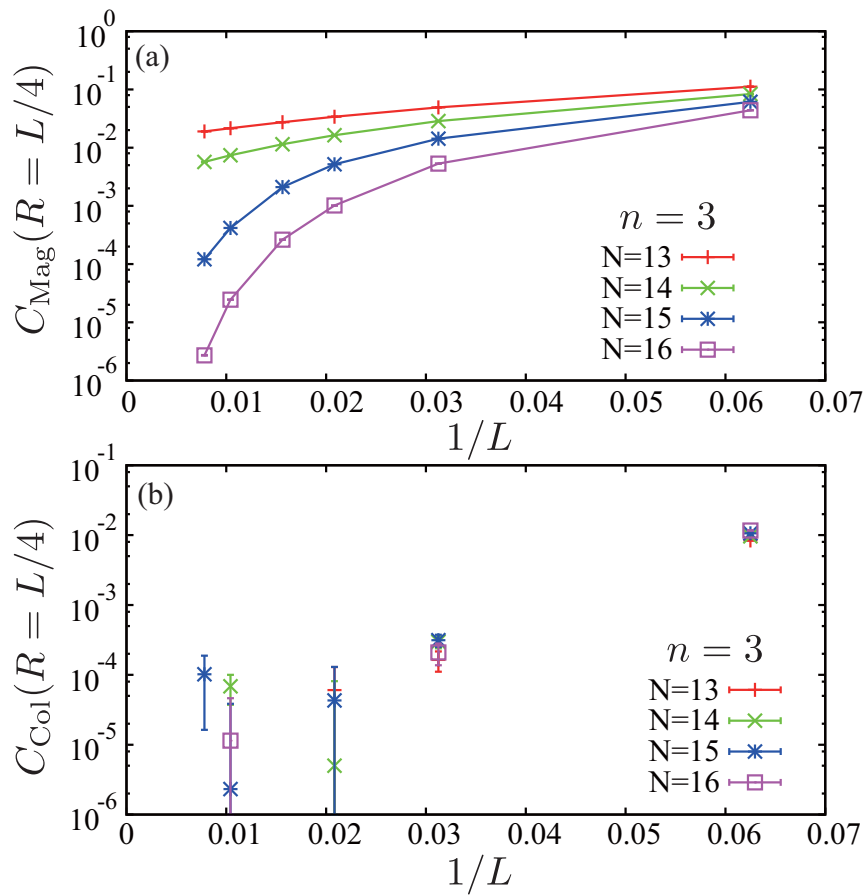

FIG. 4. (Color online) Semilog plot of the two-point correlation functions at $|\boldsymbol{R}|=L / 4$ for the model with $n=3$ as functions of $1 / L$. (a) The correlation function of the Néel order. (b) The correlation function of the columnar VBS order. The error bars represent the standard errors.

parameters may indicate that the VBS order is too small to be visible, even if it exists for $L \leqslant 128$ finite systems with the present statistical errors. ${ }^{2}$ Indeed, based on the $1 / N$ expansion, Read and Sachdev proposed that the amplitude of the VBS order parameter becomes exponentially small by increasing $N$ as $\left|\left\langle\Psi_{j}\right\rangle\right| \sim \exp \left(-N E_{c}\right)$ with the action of a hedgehog instanton [1]. The constant $E_{c}$ has been calculated as $E_{c}=c \ln \xi$ with $c=0.12459 \ldots$ in the limit $N \rightarrow \infty$ with the spin correlation length $\xi$ large but fixed [2].

By using the result of the large $N$ theory [1], we try to estimate the expected amplitude of the VBS order. For the columnar VBS order parameter, a more precise expression for our definition of $\Psi_{j}$ is given by

$$
\left|\left\langle\Psi_{j}\right\rangle\right|=\frac{N a}{\sqrt{2}} \exp \left(-N E_{c}\right),
$$

where $a$ is an unknown constant depending on $n / N$. For the nematic VBS order parameter we also obtain

$$
\left|\left\langle\Phi_{j}\right\rangle\right|=\frac{N a}{2} \exp \left(-N E_{c}\right) .
$$

We focus on the expected phase boundary of the columnar VBS phase $N=15$ with $n=3$. The spin correlation length at this parameter is calculated as $\xi \simeq 5.2$ from a fitting of the

\footnotetext{
${ }^{2}$ We also investigated the model with $n=3, N \leqslant 25$ at lower temperatures up to $\beta J=12 L$ for $L \leqslant 64$. However, we did not see a clear difference from the case of $\beta J=L$, and there was no visible evidence of the columnar VBS order.
} 


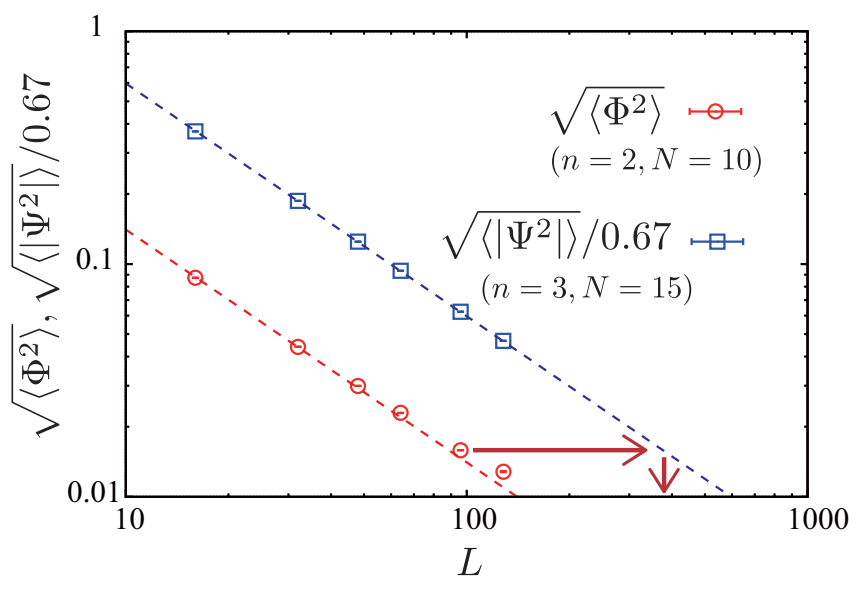

FIG. 5. (Color online) Log-log plot of the size dependence of the VBS order parameters for $(n, N)=(2,10)$ and $(n, N)=(3,15)$. The dashed lines represent the fitting curve assuming $1 / L$ decay. Arrows indicate the correspondence between $n=2$ and $n=3$ cases. The error bars represent the standard errors, which are very small compared with the symbol.

correlation function of the Néel order. In the same ratio of $n / N=0.2$, the spin correlation length at $N=10$ with $n=2$ is estimated as $\xi \simeq 4$.7. By substituting the values of $\xi$ and $N$ into two equations (6) and (7) with $E_{c} \simeq 0.12459 \ln \xi$, we obtain a relation

$$
\left|\left\langle\Psi_{j}\right\rangle_{N=15, n=3}\right| \simeq 0.67\left|\left\langle\Phi_{j}\right\rangle_{N=10, n=2}\right| .
$$

In Fig. 5, we plot the system size dependence of the columnar VBS order parameter $\sqrt{\left\langle|\Psi|^{2}\right\rangle}$, where $\Psi \equiv L^{-2} \sum_{j} \Psi_{j}$, along with that of the nematic VBS order parameter $\sqrt{\left\langle\Phi^{2}\right\rangle}$. These order parameters are expected to converge into $\left|\left\langle\Psi_{j}\right\rangle\right|$ and $\left|\left\langle\Phi_{j}\right\rangle\right|$, respectively, in the thermodynamic limit. For the purpose of better comparison, we divide $\sqrt{\left\langle|\Psi|^{2}\right\rangle}$ by the factor 0.67 which appeared in Eq. (8). In the case of $\sqrt{\left\langle|\Psi|^{2}\right\rangle}$ it decreases with $L^{-1}$, as expected for the case of no long-range order, while the $L$ dependence of $\sqrt{\left\langle\Phi^{2}\right\rangle}$ changes from $L^{-1}$ around $L \simeq 100$, indicating development of a weak long-range nematic VBS order. From comparison between $\sqrt{\left\langle|\Psi|^{2}\right\rangle}$ and $\sqrt{\left\langle\Phi^{2}\right\rangle}$, we expect that a signature of the columnar VBS order for $n=3, N=15$ becomes visible for the systems size larger than $L \simeq 400$. Therefore the fact that we did not observe any evidence of the long-range VBS order in the present calculation up to $L=128$ does not necessarily indicate the presence of an intermediate phase where both the Nèel and the VBS order disappears. Because the QMC calculation for $L \simeq 400$ requires a larger computational cost than the available resources, we cannot reach a clear answer for the phase boundary in the case of $n=3$.

\section{SUMMARY}

In summary, we have investigated the ground-state property of an $\mathrm{SU}(N)$ symmetric antiferromagnetic Heisenberg model on the two-dimensional square lattice for the representations with the $n=2$ and $n=3$ column Young diagrams. For $n=2$, we conclude that the Néel state is the ground state for $N \leqslant 9$, while the nematic VBS state becomes the ground state for $N \geqslant 10$. Thus there is no intermediate state between them [see Fig. 1(a)]. For $N<10$, we could induce the quantum phase transition between the Néel phase and the nematic VBS phase by introducing additional many-body interactions such as the $Q$ term in the $J-Q$ model [11-13,16-18]. In the theory of the deconfined critical phenomena, the nematic VBS is governed by the $Z_{2}$ symmetry-breaking field, and its irrelevance is a necessary condition for the presence of the deconfined critical phenomena [4]. Because the $Z_{2}$ symmetry-breaking field is expected to be irrelevant for $N \geqslant 4$ in noncompact $\mathrm{CP}^{N-1}$ field theories $[17,18]$, the direct transition between the Néel phase and the nematic VBS phase indicates possible deconfined critical phenomena when we vary the Hamiltonian parameters continuously, such as the $J-Q$ model [11-13,16-18]. For $n=3$, the ground state for $N \leqslant 14$ is the Néel state and it disappears for $N \geqslant 15$. Although we observed no evidence of the expected columnar VBS order for $N \geqslant 15$, this observation does not exclude the columnar VBS order in this case, because we estimated that the signature of the VBS order was invisible for smaller sizes $L \lesssim 400$, even if it eventually converges to a finite value. Determining the VBS phase boundary for $n \geqslant 3$ requires further studies. Our analysis indicates that we need careful extrapolations of the finite-size data into the thermodynamic limit. Naive extrapolations may lead to an incorrect characterization of the intermediate region even if a weak VBS order is eventually stabilized [22].

\section{ACKNOWLEDGMENTS}

We owe thanks for helpful discussions to $\mathrm{S}$. Todo, $\mathrm{T}$. Suzuki, H. Watanabe, and H. Matsuo. The computation in the present work was partly executed on computers at the Supercomputer Center, ISSP, University of Tokyo. The present work is financially supported by JSPS KAKENHI Grants No. 25287097 and No. 26400392, and by CMSI, MEXT-SPIRE, Japan.
[1] N. Read and S. Sachdev, Phys. Rev. B 42, 4568 (1990).

[2] N. Read and S. Sachdev, Nucl. Phys. B 316, 609 (1989).

[3] T. Senthil, A. Vishwanath, L. Balents, S. Sachdev, and M. P. A. Fisher, Science 303, 1490 (2004).

[4] T. Senthil, L. Balents, S. Sachdev, A. Vishwanath, and M. P. A. Fisher, Phys. Rev. B 70, 144407 (2004).
[5] T. Senthil, L. Balents, S. Sachdev, A. Vishwanath, and M. P. A. Fisher, J. Phys. Soc. Jpn. 74, 1 (2005).

[6] K. Harada, N. Kawashima, and M. Troyer, Phys. Rev. Lett. 90, 117203 (2003)

[7] N. Kawashima and Y. Tanabe, Phys. Rev. Lett. 98, 057202 (2007).

[8] K. S. D. Beach, F. Alet, M. Mambrini, and S. Capponi, Phys. Rev. B 80, 184401 (2009). 
[9] F. Haldane, Phys. Lett. A 93, 464 (1983).

[10] F. D. M. Haldane, Phys. Rev. Lett. 50, 1153 (1983).

[11] A. W. Sandvik, Phys. Rev. Lett. 98, 227202 (2007).

[12] J. Lou, A. W. Sandvik, and N. Kawashima, Phys. Rev. B 80, 180414 (2009).

[13] R. G. Melko and R. K. Kaul, Phys. Rev. Lett. 100, 017203 (2008).

[14] A. B. Kuklov, M. Matsumoto, N. V. Prokof'ev, B. V. Svistunov, and M. Troyer, Phys. Rev. Lett. 101, 050405 (2008).

[15] R. K. Kaul and A. W. Sandvik, Phys. Rev. Lett. 108, 137201 (2012).

[16] K. Chen, Y. Huang, Y. Deng, A. B. Kuklov, N. V. Prokof'ev, and B. V. Svistunov, Phys. Rev. Lett. 110, 185701 (2013).
[17] K. Harada, T. Suzuki, T. Okubo, H. Matsuo, J. Lou, H. Watanabe, S. Todo, and N. Kawashima, Phys. Rev. B 88, 220408 (2013).

[18] M. S. Block, R. G. Melko, and R. K. Kaul, Phys. Rev. Lett. 111, 137202 (2013).

[19] S. Todo and K. Kato, Phys. Rev. Lett. 87, 047203 (2001).

[20] B. Bauer, L. D. Carr, H. G. Evertz, A. Feiguin, J. Freire, S. Fuchs, L. Gamper, J. Gukelberger, E. Gull, S. Guertler, A. Hehn, R. Igarashi, S. V. Isakov, D. Koop, P. N. Ma, P. Mates, H. Matsuo, O. Parcollet, G. Pawowski, J. D. Picon et al., J. Stat. Mech.: Theory Exp. (2011) P05001.

[21] S. Todo, H. Matsuo, and H. Shitara (unpublished).

[22] A. W. Sandvik, Phys. Rev. B 85, 134407 (2012). 